\section{Ampliación del rango de distribución de Rhipidomys leucodactylus (Rodentia: Cricetidae): una nueva localidad para el suroriente del Ecuador}

\section{Carlos Nivelo-Villavicencio ${ }^{* *}$, Javier Fernández de Córdova ${ }^{1,2}$, Pedro X. Astudillo³}

'Laboratorio de Mastozoología, Museo de Zoología de la Universidad del Azuay, Av. 24 de mayo 7-77 y Hernán Malo. Cuenca, Ecuador.

2Facultad de Ciencias Exactas, Universidad de Buenos Aires, Av. Int. Güiraldes 2620, Pabellón II, Ciudad Universitaria, 1428 Ciudad Autónoma de Buenos Aires, Argentina

¿Laboratorio de Ecología, Escuela de Biología, Ecología y Gestión, Universidad del Azuay, Av. 24 de mayo 7-77 y Hernán Malo. Cuenca, Ecuador.

*Autor principal/corresponding autor, e-mail: cvillavicencio@uazuay.edu.ec

\section{Extension of the distribution range of Rhipidomys leucodactylus (Rodentia: Cricetidae): a new locality in south-eastern Ecuador}

\section{Abstract}

Rhipidomys leucodactylus has been reported from the Andean slopes of western and northeastern Ecuador. Herein, we report an adult male R. leucodactylus from a low montane evergreen forest on the southeastern Andes of Ecuador, at an elevation of $2164 \mathrm{~m}$. This report provides the highest known record of the species and a new locality in southeastern Ecuador.

Keywords: Andes, distribution, elevation, Morona-Santiago, Sigmodontinae, TinajillasRío Gualaceño.

\section{Resumen}

Rhipidomys leucodactylus ha sido reportado en las laderas andinas del occidente $y$ nororiente del Ecuador. En este trabajo nosotros reportamos un macho adulto de $R$. leucodactylus proveniente de un bosque siempre verde montano bajo al sureste de los Andes de Ecuador, a una altitud de $2164 \mathrm{~m}$. Este reporte proporciona el registro más alto conocido para la especie y una nueva localidad en el sureste de Ecuador.

Palabras clave: Andes, distribución, elevación, Morona Santiago Sigmodontinae Tinajillas-Río Gualaceño

\section{INTRODUCCION}

El género Rhipidomys (Cricetidae: Sigmodontinae: Thomasomyni) cuenta actualmente con 23 especies distribuidas desde la península de Paraguaná en Venezuela y la parte oriental de Panamá hasta la provincia de Salta al noroeste de Argentina [1-2]. A lo largo de su distribución ocupa diferentes hábitats, desde bosques húmedos de tierras bajas hasta bosques alto andinos cerca del páramo [2-3]. En Ecuador, se han reportado dos especies del género Rhipidomys: la Rata Trepadora de Pies Blancos Rhipidomys leucodactylus (Tschudi, 1845) y la Rata Trepadora de Pies Anchos R. latimanus (Tomes, 1860) [2,4-5]. Rhipidomys leucodactylus se encuentra distribuida en las Guayanas, sur de Venezuela, norte y centro de Brasil, Ecuador, Perú y en el centro oeste de Bolivia [3-4]. En Ecuador ocurre en los bosques húmedos de tierras bajas del Pacífico, los bosques piemontanos de la Cordillera Occidental de los Andes, los bosques piemontanos y montanos de la Cordillera Oriental de los Andes, y los bosques húmedos de tierras bajas de la Amazonía, entre 200 y $1600 \mathrm{~m}$ [6] A pesar de su amplio rango de distribución, pocos registros se han reportado, incluyendo: San José, provincia de Orellana; Mera, provincia de Pastaza; Palora, provincia de Morona Santiago; Los Pozos, provincia de El Oro; Santo Domingo, provincia de Santo Domingo de los Tsáchilas; y, Achiotes y El Faique, provincia de Loja [2,7-8]. En esta publicación presentamos un nuevo registro de R. leucodactylus para el suroriente del Ecuador, que constituye la localidad a mayor altitud conocida para la especie.

El 24 de mayo de 2014, se registró un macho adulto de R. leucodactylus en el Área de Conservación Municipal Tinajillas-Río Gualaceño $\left(3.00^{\circ} \mathrm{S}, 78.49^{\circ} \mathrm{W} ; 2164 \mathrm{~m}\right)$, cantón Limón-Indanza, provincia de Morona Santiago. El espécimen fue hallado muerto en uno de los senderos que atraviesa un bosque montano alto primario de estribación, piso zoogeográfico Templado [9], estribación suroriental de la Cordillera de los Andes (Fig. 1). Este registro se realizó durante una investigación de anfibios, el espécimen posteriormente fue llevado al Museo de Zoología de la Universidad del Azuay (MZUA), donde fue preparado y depositado dentro de la colección mastozoológica con el código MZUA-MA171.

Algunos de los caracteres morfológicos externos que identifican al género Rhipidomys son: patas posteriores anchas con almohadillas plantares desarrolladas, un conjunto de pelos oscuros en el dorso; cola gruesa y peluda, a menudo con un mechón de pelos terminales de longitud variable; rostro corto con ojos grandes y vibrisas mistaciales largas que sobrepasan las puntas de las orejas cuando se extienden hacia atrás [1]. A continuación se describen las principales características morfológicas y las medidas morfométricas se presentan en la Tabla 1. El espécimen es de tamaño grande y contextura robusta, el pelaje dorsal es un poco áspero de color amarillo rojizo con la base gris oscura y algunos pelos de color negro entremezclados, en la región ventral el pelo es de color blanco con la base gris pálida, en no más de $3 \mathrm{~mm}$. Las orejas son evidentes $y$ desnudas, con pelos de color ocre alrededor de la base. La cola es larga, sobrepasa $120 \%$ de la longitud de la cabeza y el cuerpo juntos, es de color negro uniforme con abundante pelaje y en la punta tiene un penacho que alcanza los $30 \mathrm{~mm}$ (Fig. 2C). Las patas posteriores son largas (Fig. 2A) y las anteriores cortas (2B), presentan almohadillas carnosas y desarrolladas que son características relacionadas con sus hábitos arborícolas [11], el pelaje dorsal de las patas traseras es negro y se torna blancuzco hacia la punta de los dedos (Fig 2C). El cráneo es bastante robusto y con las crestas supraorbitales notorias (Fig. 3A-C), los incisivos superiores son de color anaranjado en la parte frontal (Fig. 3B) y 
aci

los inferiores presentan un tono más claro. El cóndilo mandibular apenas sobrepasa la apófisis angular (Fig. 3D) y con la hileras maxilares superior e inferior largas en relación al cráneo (Fig. 3E-F). Las características de este espécimen coinciden con las referidas en Mammals of South America Volume 2 Rodents [2] dentro de rangos fenotípicos de coloración, forma y tamaño para la especie.

Rhipidomys latimanus, difiere externamente de R. leucodactylus por ser menos grande y robusta, con la cola bicolor y con un penacho usualmente de $10 \mathrm{~mm}$ (cola de color uniforme y con un penacho bien desarrollado $40 \mathrm{~mm}$ en R. latimanus), y por no mostra el pelaje dorsal de las patas blanquecino hacia la punta de los dedos (particularidad que sucede en $R$. leucodactylus). Rhipidomys gardneri, especie morfológicamente similar a R. leucodactylus, difiere por la coloración del pelaje ventral amarillento y con la base gris hacia la mitad de los pelos de la garganta y el pecho (pelaje ventral blanco en $R$ leucodactylus) y por el penacho de la cola no más allá de $6 \mathrm{~mm}$ [12].

Este registro extiende $\sim 414 \mathrm{~m}$ el límite altitudinal superior de la especie, pues previamente la máxima altitud se había registrado hasta los $1750 \mathrm{~m}$ al este de Perú [2]. En el territorio ecuatoriano este espécimen representa una nueva localidad para la provincia de Morona Santiago y amplía el rango de distribución al sur oriente del país.

\section{AGRADECIMIENTOS}

A Verónica Urgilés y Cristian Nieves por realizar la colecta del individuo. A Juan Carlos Sánchez por su colaboración con la fotografías del cráneo y a la Universidad del Azuay por el apoyo logístico e infraestructura. A Jorge Brito por la información y revisión de espécimen. Este estudio fue apoyado por los fondos de investigación FONDOS UDA 2014 (Código 2014-08). El Museo de Zoología de la Universidad del Azuay está regido por la patente ambiental FAUS-UDA-MUSEO DE ZOOLOGÍA-003-2016.

TABLA 1. Medidas morfométricas del espécimen macho de Rhipidomys leucodactylus (MZUA-MA171) registrado en la localidad de Tinajillas-Rio Gualaceno, provincia de Morona Santiago. Medidas tomadas con un calibrador digital marca Truper de $200 \mathrm{~mm}$, siguiendo las especificaciones de Expedition Field Techniques [10] Medidas promedio de la especie tomadas de Mamiferos del Ecuador [6].

\begin{tabular}{|lcc|}
\hline \multicolumn{1}{c}{ Medida } & $\begin{array}{c}\text { Medidas (en mm) } \\
\text { Espécimen MZUA-MA171 }\end{array}$ & $\begin{array}{c}\text { Medidas (en mm) promedio } \\
\text { para R. leucodactylus }\end{array}$ \\
\hline Largo cabeza cuerpo & 210 & $136-192$ \\
\hline Largo cola & 264 & $176-230$ \\
\hline Largo oreja & 22 & $17-23$ \\
\hline Largo pata posterior (con uña) & 37 & $29-38$ \\
\hline Constricción interorbital & 6,1 & - \\
\hline Hilera maxilar superior & 6,9 & - \\
\hline Longitud de los huesos nasales & 16,2 & - \\
\hline Ancho cigomático & 23,3 & - \\
\hline Longitud orbital & 13,6 & - \\
\hline
\end{tabular}

\begin{tabular}{lcc} 
Longitud diastema & 11,6 & - \\
\hline Longitud foramen incisivo & 7,8 & - \\
\hline Largo cráneo & 43,1 & - \\
\hline Ancho cráneo & 17,2 & -
\end{tabular}

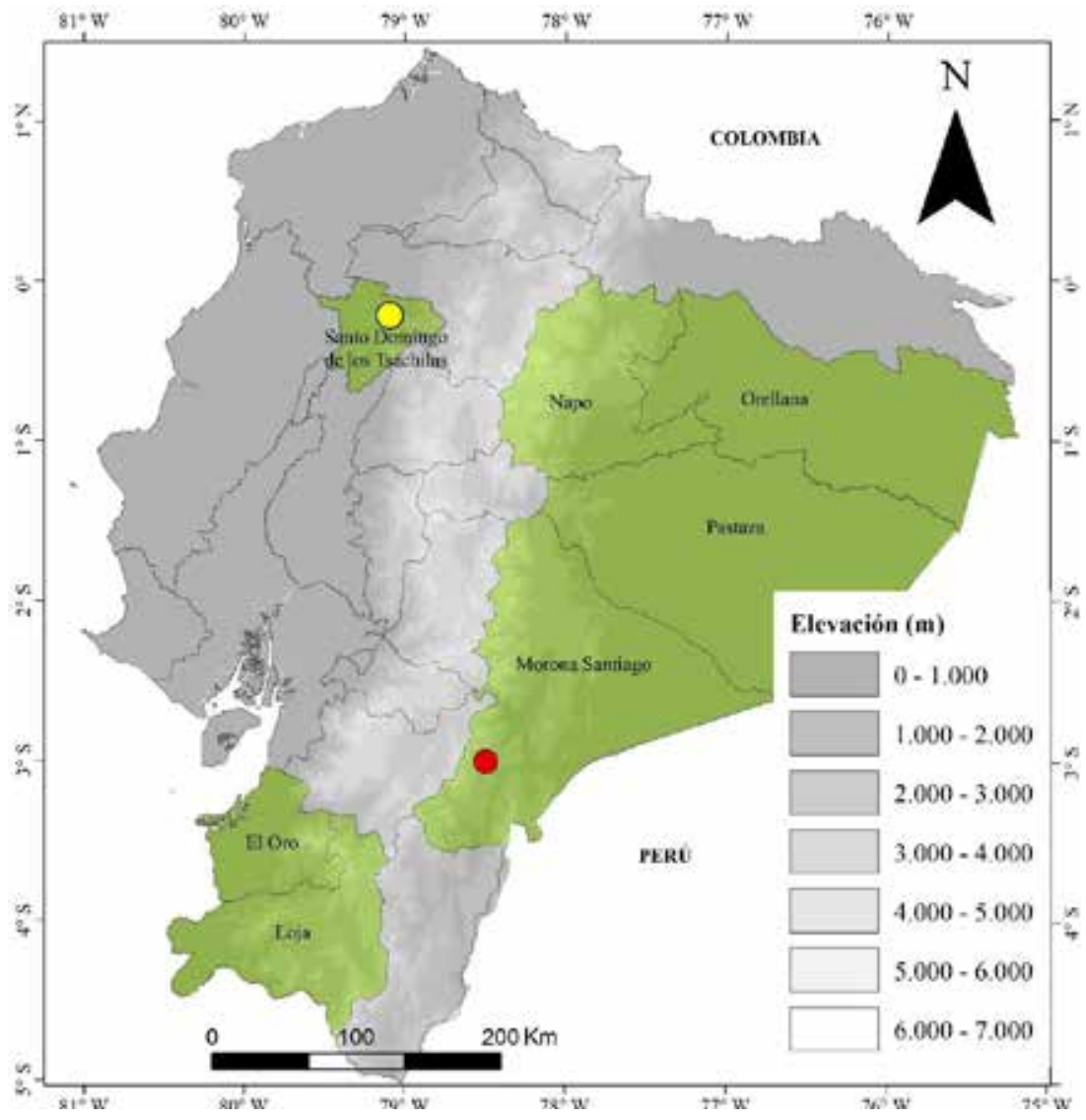

FIGURA 1. Distribución de R. leucodactylus en el Ecuador. En verde las provincias donde se ha reportado a la especie. El circulo amarillo representa a $R$. equatoris, sinonimo de R. leucodacty/us, a $1600 \mathrm{~m}$ en la provincia de Santo Domingo de los Tsáchilas [2]. El círculo rojo representa la nueva localidad aquí reportada en Tinajillas-Río Gualaceño, provincia de Morona Santiago. 


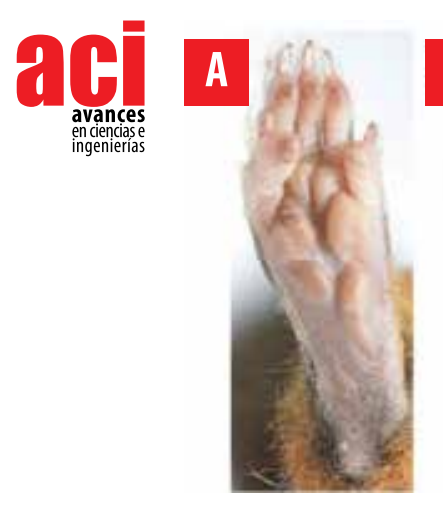

B

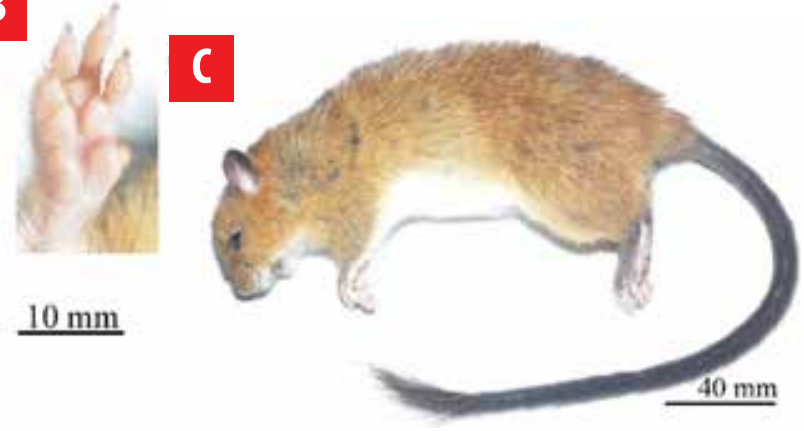

FIGURA 2. Espécimen macho adulto de Rhipidomys leucodactylus (MAZUA-MA171) registrado en la localidad de Tinajillas-Río Gualaceño, provincia de Morona Santiago. A) vista plantar de la pata posterior derecha, B) vista plantar de la pata anterior derecha, () vista lateral del individuo.

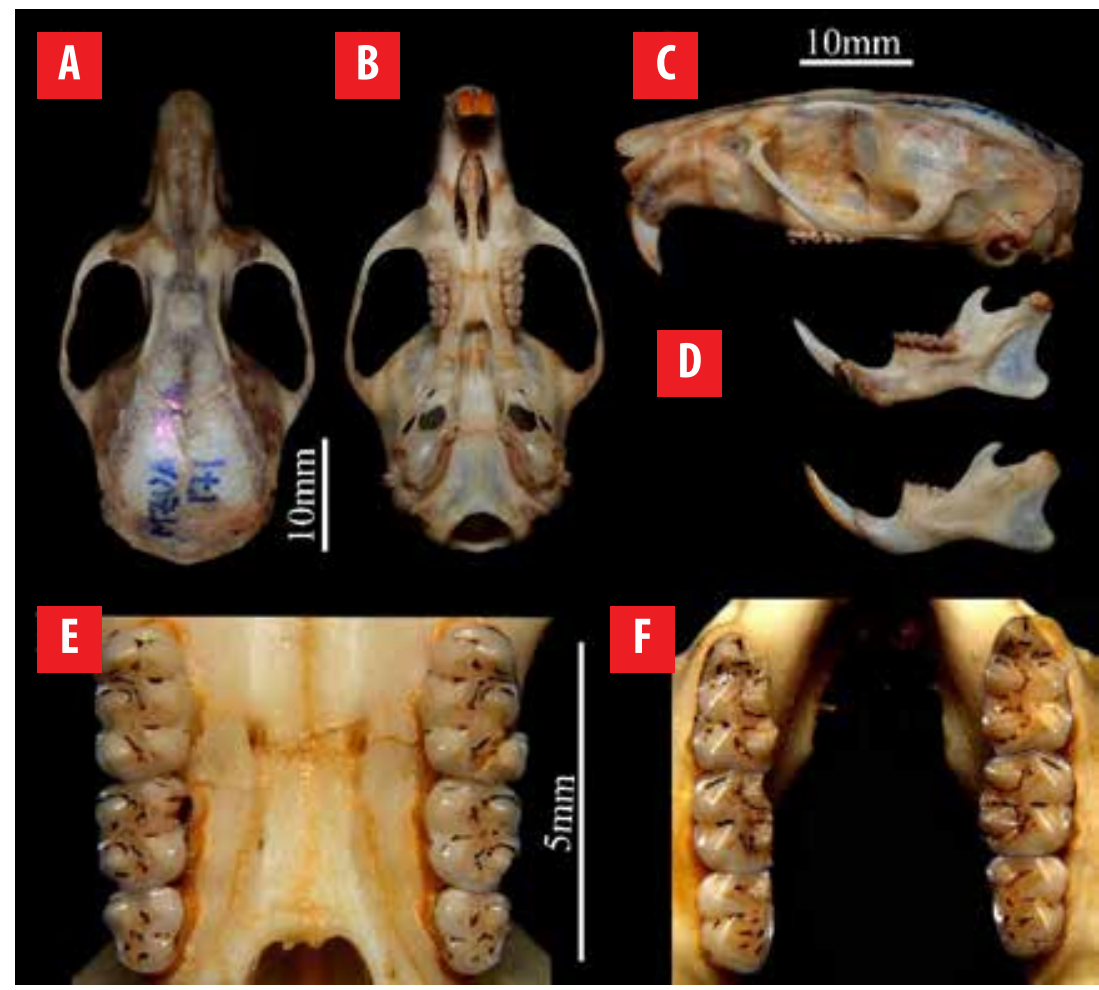

FIGURA 3. Cráneo del espécimen macho adulto de Rhipidomys leucodactylus (MAZUA-MA171) registrado en la localidad de Tinajillas-Rio Gualaceño, provincia de Morona Santiago. A) Vista dorsal, B) Vista ventral, () Vista lateral, D) Vista lateral interna y externa de la mándíbula derecha, E) Vista de los molares superiores, F) Vista de los molares inferiores.

\section{REFERENCIAS}

[1] Voss, R. S., Lunde, D. P. \& Simmons, N. B. (2001). The mammals of Paracou, French Guiana: a neotropical lowland rainforest fauna part 2. Nonvolant species. Bulletin of the American Museum of Natural History, 3-236.

[2] Patton, J. L., Pardiñas, U. F., \& D'Elía, G. (Eds.). (2015). Mammals of South America, volume 2: Rodents. Vol. 2. University of Chicago Press.

[3] Tribe, C. J. (2005). A new species of Rhipidomys (Rodentia, Muroidea) from north- eastern Brazil.Arquivos do Museu Nacional, Rio de Janeiro, 63(1), 131-146.

[4] Musser, G. G., \& Carleton, M. D. (2005). Subfamily Sigmodontinae. Mammal species of the world, a taxonomic and geographic reference, $3,1086-1185$.

[5] Tirira, D. G. (2016). Mamifferos del Ecuador: lista actualizada de especies / Mammals of Ecuador: Updapted checklist species. Versión 2016.1. Asociación Ecuatoriana de Mastozoología y Fundación Mamíferos y Conservación. Quito. (actualización / updated: 2016-07-12). doi: 10.13140/RG.2.1.1508.2489

[6] Tirira, D. (2007). Guía de campo de los mamíferos del Ecuador. Publicación Especial sobre los Mamíferos del Ecuador 6. Quito: Ediciones Murciélago Blanco.

[7] Brito, J., \& 0jala-Barbour, R. (2016). Mamiferos no voladores del Parque nacional sangay, ecuador. Papéis Avulsos de Zoologia (São Paulo), 56(5).

[8] Boada, C., \& Román, H. (2005). Evaluación ecológica rápida de la mastofauna en dos localidades de bosque seco en el occidente de la provincia de Loja. Biodiversidad. En, M.A. Vázquez, J.F. Freire \& L. Suárez (Eds.).Los Bosques Secos de la Zona de Cerro Negro-Cazaderos, al Occidente de la Provincia de Loja: Un Reporte de las Evaluaciones Ecológicas y Socioeconómicas Rápidas. Ecociencia, MAE y Proyecto Bosque Seco. Quito-Ecuador, 73-90.

[9] Albuja, L. (2011). Lista de mamiferos actuales del Ecuador. Instituto de Ciencias Biológicas, Escuela Politécnica Nacional. Casilla 17-01-2759, Quito.

[10] Barnett, A., \& Dutton, J. (1995). Small mammals.Expedition Field Techniques series, Expedition Advisory Centre, Royal Geographical Society. London.

[11] de Camargo, N. F., Gonçalves, R. G., \& Palma, A. R. T. (2008). Variação morfológica de pegadas de roedores arborícolas e cursoriais do Cerrado. Zoologia, 25(4)

[12] Patton, J. L., Da Silva, M. N. F., \& Malcolm, J. R. (2000). Mammals of the Rio Juruá and the evolutionary and ecological diversification of Amazonia. Bulletin of the American Museum of Natural History, 1-306.

\section{CONTRIBUCIONES DE LOS AUTORES}

Carlos Nivelo-Villavicencio, Javier Fernández de Córdova y Pedro X. Astudillo revisaron e identificaron el espécimen, revisaron los registros de distribución, literatura especializada y escribieron el manuscrito. 